\title{
AVALIAÇÃO DOS NÍVEIS DE PRESSÃO ARTERIAL E FREQUÊNCIA CARDÍACA E DISLIPIDEMIAS EM ESCOLARES COM PERSPECTIVA NA PROMOÇÃO DA SAÚDE E PREVENÇÃo PRIMÁRIA
}

\author{
Larissa de Matos Souza $^{1}$; Érica Maria Granjeiro²; Aline Moraes Bergossi Gome ${ }^{3}$, \\ Joelande Esquivel Correia ${ }^{4}$ \\ 1. Bolsista PROBIC/UEFS, Graduanda em Enfermagem, Universidade Estadual de Feira de Santana, e-mail: \\ larissa.uefs@gmail.com \\ 2. Orientadora, Coordenadora do Grupo de Pesquisa em Ensino de Fisiologia (GPFISIO), Departamento de Ciências \\ Biológicas, Universidade Estadual de Feira de Santana, e-mail: ericag@uefs.br \\ 3. Pesquisadora do Grupo de Zoonoses e Saúde Pública da UEFS e colaboradora do GPFISIO, Departamento de Ciências \\ Biológicas, Universidade Estadual de Feira de Santana, e-mail: alinemoraesx @ gmail.com \\ 4. Pesquisadora do Grupo de Zoonoses e Saúde Pública da UEFS e colaboradora do GPFISIO, Departamento de Ciências \\ Biológicas, Universidade Estadual de Feira de Santana, e-mail: joelandecorreia@ gmail.com
}

PALAVRAS-CHAVE: hipertensão arterial, escolares, dislipidemias.

\section{INTRODUÇÃO}

A discussão sobre hábitos alimentares das crianças e a prevenção das principais lesões cardiovasculares têm ganhado destaque na atualidade (PAES et al., 2015). "As dislipidemias e a obesidade na infância e na adolescência adquiriram maior importância entre as patologias mais estudadas neste século pelas repercussões que trazem na vida adulta" (FAULHABER et al., 2009 p.4), principalmente no que se refere às doenças cardiovasculares. Atualmente, encontramos uma sociedade na qual os indivíduos não conseguem incorporar informações, valores, atitudes e habilidades que lhes permitam adotar um estilo de vida ativo e saudável, por deficiências em sua formação educacional. Nesse contexto, estabelecer estratégias de promoção da saúde e prevenção primária apresenta-se como uma proposta pertinente e desafiadora. Diante dessa problemática, o presente estudo teve como objetivo analisar a prevalência de HDL baixo $(\leq 35 \mathrm{mg} / \mathrm{dL})$ em escolares e a correlação desta com outros fatores de risco cardiovasculares e estilo de vida, bem como realizar atividades de cunho educativo em uma comunidade escolar.

\section{MATERIAL E MÉTODOS}

A pesquisa foi aprovada pelo Comitê de Ética em Pesquisa com Seres Humanos da Universidade Estadual de Feira de Santana sob número 1.524.897 e desenvolvida pelo Grupo de Pesquisa em Ensino de Fisiologia em parceria com o Grupo de Zoonoses e Saúde Pública da UEFS. Foram incluídos 160 estudantes com idade entre sete e 17 anos de uma escola pública de Feira de Santana, Bahia. Foram medidos os níveis plasmáticos de triglicerídeos e níveis basais de pressão arterial e frequência cardíaca, além de preenchido questionários relacionados à saúde comportamentos preventivos, sedentarismo, percepção sobre a condição e prioridade da saúde. Os questionários foram baseados no estudo de Azumbuja et al. (2014). Ademais, com base no trabalho de Granjeiro (2016) foram realizadas atividades de cunho educativo incluindo palestras, seminários, debates, teatros e construção de murais educativos.

Os resultados estão apresentados em valores absolutos, média \pm erro padrão da média (EPM) e valores percentuais (\%). Para comparações entre os grupos utilizou-se o Teste t de Student não pareado. O nível de significância adotado foi de $\mathrm{p} \leq 0,05$. 


\section{ANÁLISE E DISCUSSÃO DOS RESULTADOS}

No que se refere ao comportamento dos estudantes relacionados à saúde, como podemos observar na Figura 1, 131 estudantes $(81,8 \%$ da população de estudantes avaliados) relataram que não verificavam a pressão arterial com regularidade; $60 \%$ não realizavam testes bioquímicos frequentemente, $53 \%$ não controlavam os alimentos ingeridos regularmente e $61,3 \%$ relataram sedentarismo.

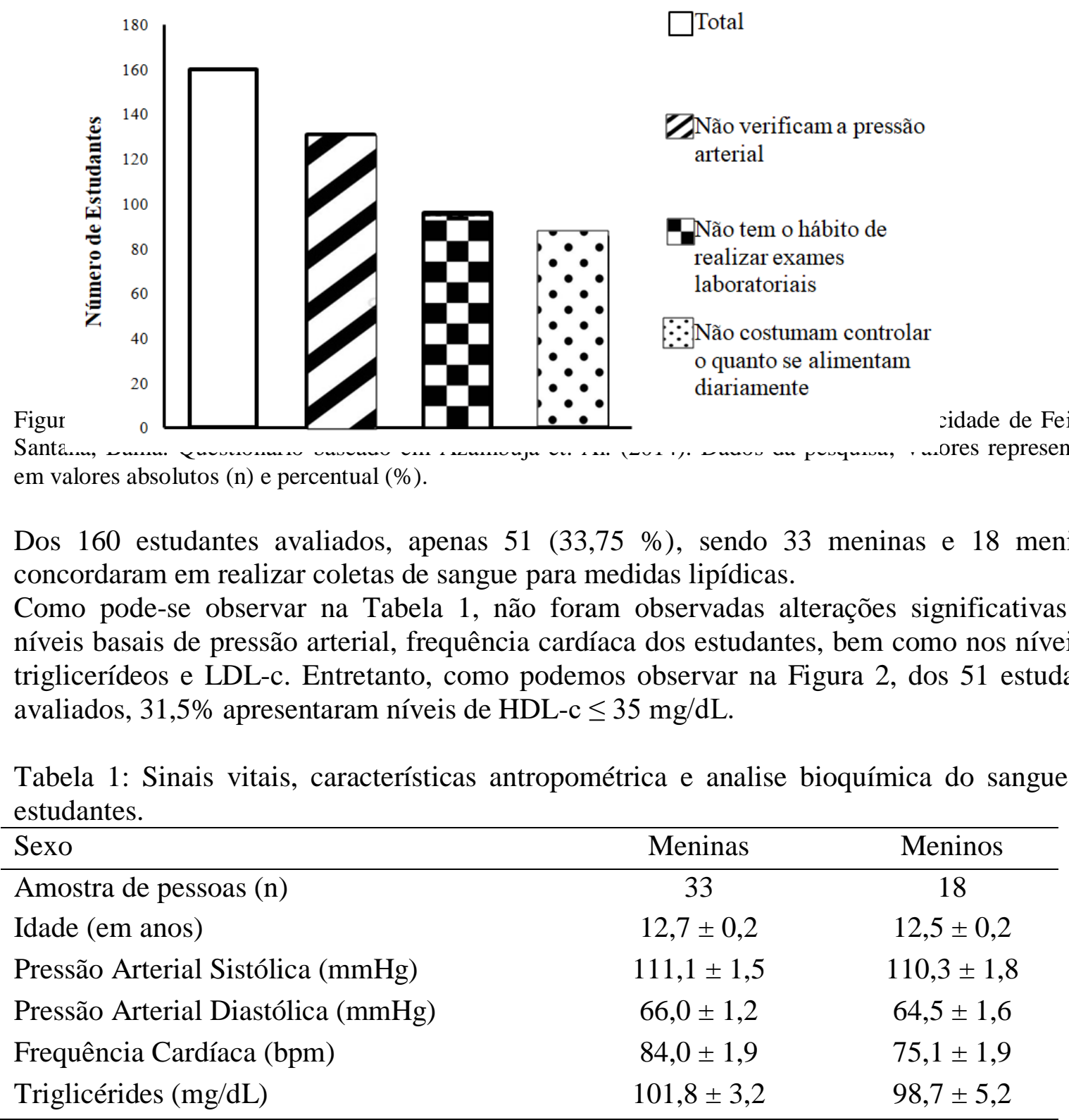

\footnotetext{
Legenda: Valores basais de Pressão Arterial Sistólica e Diastólica (mmHg), Frequência Cardíaca (bpm) e níveis de triglicérides $(\mathrm{mg} / \mathrm{dL})$ dos estudantes. Valores como média \pm erro padrão da média (EPM)
}

Com relação às atividades de educação em saúde realizadas na escola, $72 \%$ dos estudantes relataram entusiasmo em adotar e/ou implementar novos hábitos de vida saudáveis. 


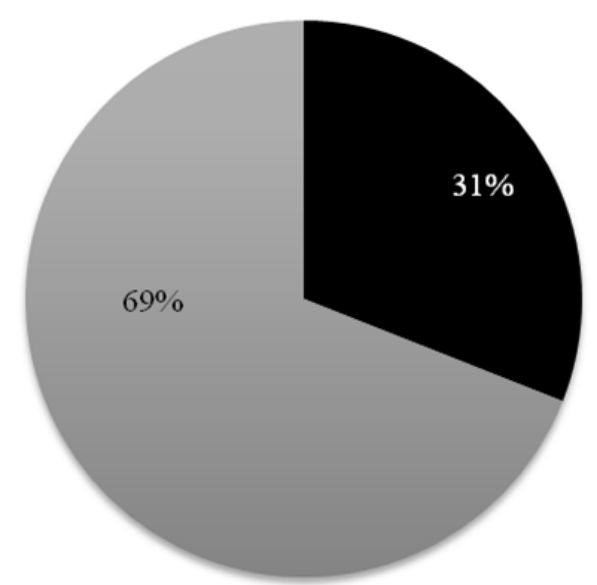

Figura: 2. Valores em \% HDL-C dos estudantes. Número total de estudantes avaliados ( $\mathrm{n}=51)$.

A dislipidemia, níveis anormal dos lipídicos no sangue, é um importante fator de risco para doenças cardiovasculares e acidente vascular cerebral - principal causa global de mortalidade (MUSUNURU et al., 2010). As doenças cardiovasculares (DCV), juntamente com outras doenças não transmissíveis (NCDs) são responsáveis por $70 \%$ das mortes no mundo (WORLD HEALTH ORGANIZATION, 2017). Segundo a World Health Organization (2015), as mortes prematuras por NCDs podem ser significativamente reduzidas por meio de políticas públicas voltadas para a redução dos fatores modificáveis como políticas voltadas para a redução do uso de tabaco, o uso prejudicial de álcool, dietas não saudáveis e inatividade física, além de oferecer assistência universal à saúde.

Em suma, as doenças cardiovasculares causam milhões de mortes por ano em todo o mundo e poderiam ser evitadas com medidas preventivas. Nesse contexto, no presente estudo, na Escola Básica, além das pesquisas laboratoriais e comportamentais, várias atividades de Educação em Saúde foram propostas direcionadas à prevenção das doenças cardiovasculares, principalmente, no que diz respeito à Hipertensão Arterial Sistêmica. As atividades de educação em saúde realizadas, no presente estudo, por meio de um processo educativo participativo proporcionaram ações de trabalho interdisciplinar, envolvendo estudantes de diferentes cursos de graduação em Educação e Saúde da UEFS (Licenciatura em Educação Física, Licenciatura e Bacharelado em Biologia, Medicina e Enfermagem), favorecendo as atividades de promoção de saúde e divulgação da Fisiologia tendo a escola como um espaço da atenção básica.

Segundo Barbosa et al. (2013), a inserção de estudantes da saúde incluindo médicos, enfermeiros, entre outros nas práticas educativas na promoção da saúde de escolares constituise uma ação pertinente e necessária. A partir do contato mais próximo com a realidade escolar, os discentes têm a oportunidade de desenvolver autonomia, senso crítico, reflexivo, criatividade e responsabilidade social, além da oportunidade de associar e até mesmo aplicar na prática vários conceitos adquiridos em sala de aula. No que se refere aos alunos da Escola participantes da Pesquisa, em todas as etapas do processo foi possível construir um espaço dialógico com os estudantes, já que eles tiveram a oportunidade de manifestar suas opiniões. Ademais, os resultados laboratoriais foram entregues aos pais e/ou responsáveis seguindo com encaminhamentos para as Unidades de Saúde, no caso de detecção de morbidades.

\section{CONSIDERAÇÕES FINAIS}


No presente estudo, alta porcentagem de estudantes não apresentou comportamentos preventivos à saúde, bem como níveis de atividade física mínimos para uma qualidade de vida melhor. Ademais, foi observada na população estudada, uma alta prevalência de indivíduos com HDL baixo. Considerando que a dislipidemia pode ser influenciada por modificações de estilo de vida, é necessária uma investigação mais aprofundada sobre o possível efeito da atividade física em níveis de HDL-c de adolescentes. Ademais, haja vista a importância da realização de atividades educativas e serviços de saúde direcionados a escolares, novas edições serão realizadas alcançando um maior número de escolas e alunos. Tais ações estreitando o diálogo entre Universidade e Escola, vêm ao encontro dos anseios da Educação Básica, que busca parcerias com profissionais de saúde, objetivando o desenvolvimento de trabalhos que atendam à proposta dos temas transversais, aos projetos políticos de educação em saúde, e outras demandas que a escola recebe continuamente.

\section{REFERÊNCIAS}

AZAMBUJA, C. R. et al. Educação científica: relação entre o estilo de vida e as ações preventivas de saúde em adolescentes de escolas públicas federais. Revista Ciências\&Ideias. v. 5, n.2. mai/out -2014 .

BARBOSA, E. F.; MOURA, D. G. Metodologias ativas de aprendizagem na Educação Profissional e Tecnológica. B. Tec. Senac, Rio de Janeiro, v.39, n.2, p.48-67, 2013.

FAULHABER, M.C.B.; FERNANDES M.A., ROISEMAN, M.M.L.; TAAM FILHO, W. Dislipidemias na infância e na adolescência: um caso de saúde pública?. - Revista de Pediatria SOPERJ. 2009. v.10, n. 1, p. 4-15.

GRANJEIRO, E.M. Trabalho interdisciplinar entre Universidade e Educação Básica para divulgação da Fisiologia: Relato de Experiência. Interagir: pensando a extensão, Rio de Janeiro, n. 21, p. 166-174, jan./jun. 2016.

PAES, S.T.; MARINS, J.C.B.; ANDREAZZI, A.E. Efeitos metabólicos do exercício físico na obesidade infantil: uma visão atual. Rev. Paul. Pediatr., v.33, n.1, p.122-

129. http://doi.org/10.1016/j.rpped.2014.11.002

WORLD HEALTH ORGANIZATION. Noncommunicable diseases prematurely take 16 million lives annually, WHO urges more action. News release 19 Jan. 2015. Geneva.

WORLD HEALTH ORGANIZATION. Noncommunicable Diseases Progress Monitor, 2017. Geneva, 2017. Licence: CC BY-NC-SA 3.0 IGO. 